\title{
Injectable Supramolecular Polymer-Nanoparticle Hydrogels for Cell and Drug Delivery Applications
}

\author{
Catherine M. Meis ${ }^{*}$, , Abigail K. Grosskopf ${ }^{*}$, , Santiago Correa ${ }^{1}$, Eric A. Appel ${ }^{1,3,4}$ \\ ${ }^{1}$ Department of Materials Science \& Engineering, Stanford University ${ }^{2}$ Department of Chemical Engineering, Stanford University ${ }^{3}$ Department of \\ Bioengineering, Stanford University ${ }^{4}$ Department of Pediatrics - Endocrinology, Stanford University \\ *These authors contributed equally
}

\section{Corresponding Author}

Eric A. Appel

eappel@stanford.edu

\section{Citation}

Meis, C.M., Grosskopf, A.K., Correa, S., Appel, E.A. Injectable Supramolecular Polymer-Nanoparticle Hydrogels for Cell and Drug Delivery Applications. J. Vis. Exp. (168), e62234, doi:10.3791/62234 (2021).

\section{Date Published}

February 7, 2021

DOI

$10.3791 / 62234$

URL

jove.com/video/62234

\section{Abstract}

These methods describe how to formulate injectable, supramolecular polymernanoparticle (PNP) hydrogels for use as biomaterials. PNP hydrogels are composed of two components: hydrophobically modified cellulose as the network polymer and self-assembled core-shell nanoparticles that act as non-covalent cross linkers through dynamic, multivalent interactions. These methods describe both the formation of these self-assembled nanoparticles through nanoprecipitation as well as the formulation and mixing of the two components to form hydrogels with tunable mechanical properties. The use of dynamic light scattering (DLS) and rheology to characterize the quality of the synthesized materials is also detailed. Finally, the utility of these hydrogels for drug delivery, biopharmaceutical stabilization, and cell encapsulation and delivery is demonstrated through in vitro experiments to characterize drug release, thermal stability, and cell settling and viability. Due to its biocompatibility, injectability, and mild gel formation conditions, this hydrogel system is a readily tunable platform suitable for a range of biomedical applications.

\section{Introduction}

Injectable hydrogels are an emerging tool to deliver therapeutic cells and drugs to the body in a controlled fashion ${ }^{1}$. These materials can be loaded with drugs or cells and can be administered in a minimally invasive manner through direct injection to superficial tissues or by catheter delivery to deep tissues. In general, injectable hydrogels are composed of water-swollen polymer networks that are crosslinked together by transient, physical interactions. At rest, these crosslinks provide a solid-like structure to the gels, but upon application of sufficient mechanical force these crosslinks are temporarily disrupted, transforming the material into a liquid-like state that can easily flow ${ }^{2}$. It is these rheological properties that allow physical hydrogels to shear-thin and flow through small needle diameters during injection ${ }^{3}$. After injection, the polymer network of the material reforms, allowing it to self-heal and rapidly form a solid-like gel in situ ${ }^{4,5}$. These structures can act as slow-release depots for drugs or scaffolds for tissue regeneration 6,7 . These materials 
have been used in diverse applications encompassing drug delivery technology, regenerative medicine, and immunoengineering ${ }^{1,8,9,10,11,12}$.

Both natural materials (e.g., alginate and collagen) and synthetic materials (e.g., poly(ethylene glycol) (PEG) or similar hydrophilic polymers) have been developed as biocompatible injectable hydrogel materials ${ }^{13,14,15}$. Many natural materials exhibit batch-to-batch variation affecting reproducibility ${ }^{4,16}$. These materials are often temperaturesensitive, curing upon reaching physiological temperatures; thus, handling these materials poses additional technical and logistical challenges ${ }^{17}$. Synthetic materials allow for more precise chemical control and excellent reproducibility, but these materials can sometimes be subject to adverse immune responses that limit their biocompatibility, a critical feature for in vivo therapeutic applications ${ }^{6,18,19}$. Recent efforts have shown there are many complex design criteria involved in engineering an injectable hydrogel material, including optimizing mechanical properties, polymer network mesh size, bioactive molecular cues, biodegradability, and immunogenicity of the material $20,21,22,23,24,25,26$. All of these factors must be considered depending on the application of interest, which means that a modular, chemically tunable platform is ideal for satisfying a wide breadth of applications.

The present methods describe the formulation and the use of an injectable polymer-nanoparticle (PNP) hydrogel platform that exhibits tunable mechanical properties, a high degree of biocompatibility and low immunogenicity, and presents sites for conjugating bioactive molecular cues $^{27,28,29,30,31,32,33}$. These PNP hydrogels are composed of hydrophobically-modified cellulose polymers and self-assembled core-shell nanoparticles comprising poly(ethylene glycol)-block-poly(lactic acid) (PEG-PLA) ${ }^{27,34}$ that interact to produce a supramolecular network. More specifically, the dodecyl-modified hydroxypropylmethyl cellulose polymers (HPMC- $\mathrm{C}_{12}$ ) dynamically interact with the surface of PEG-PLA nanoparticles and bridge between these nanoparticles to form this polymer network ${ }^{27,34}$. These dynamic, multivalent interactions allow the materials to shearthin during injection and rapidly self-heal after administration. The PNP hydrogel components are easily fabricated through simple one-pot reactions and the PNP hydrogel is formed under mild conditions by simple mixing of the two components $^{35}$. Due to the ease of fabrication, this hydrogel platform is highly translatable at scale. The mechanical properties and mesh size of PNP hydrogels are controlled by altering the weight percent of the polymer and nanoparticle components in the formulation. Prior studies with this platform indicate that PNP hydrogels are highly biocompatible, biodegradable, and non-immunogenic ${ }^{28,30,31}$. Overall, these hydrogels present wide utility in biomedical applications encompassing post-operative adhesion prevention, tissue engineering and regeneration, sustained drug delivery and immunoengineering.

\section{Protocol}

Prior to beginning this protocol, it is necessary to synthesize HPMC-C 12 and PEG-PLA using previously published methods $27,28,29,30,31,36,37,38$.

\section{Nanoparticle (NP) synthesis by nanoprecipitation}

NOTE: This section describes synthesis of a single batch of NPs, producing $250 \mu \mathrm{L}$ of $20 \mathrm{wt} \%$ NPs in buffer solution (50 mg of dry PEG-PLA polymer per batch). Notes for scaling up the number of batches are provided at relevant steps. 
1. Measure $50 \mathrm{mg}$ of PEG-PLA polymer into an $8 \mathrm{~mL}$ glass scintillation vial and add $1 \mathrm{~mL}$ of acetonitrile. Vortex to fully dissolve.

NOTE: To scale up the number of batches, linearly scale this step and add the total amount of polymer and solvent needed in a single vial.

2. Add $10 \mathrm{~mL}$ of ultrapure water into a $20 \mathrm{~mL}$ glass scintillation vial with a small stir bar. Put on a stir plate set to $600 \mathrm{rpm}$.

NOTE: If step 1.1 has been scaled, it is still necessary to have an individual scintillation vial to precipitate into for each equivalent batch. For example, for $200 \mathrm{mg}$ of polymer dissolved in $4 \mathrm{~mL}$ of acetonitrile, prepare $4 \times 20$ $\mathrm{mL}$ scintillation vials.

3. To form NPs by nanoprecipitation, add $1 \mathrm{~mL}$ of polymer solvent solution dropwise into the water using a $200 \mu \mathrm{L}$ pipette. Stir for $2 \mathrm{~min}$. The PLA block of the PEG-PLA is not soluble in water, and as a result, core-shell NPs will self-assemble with the hydrophobic PLA blocks as the core and the hydrophilic PEG blocks as the shell.

4. Verify the particle size by dynamic light scattering (DLS). NOTE: This procedure is specifically written for a commercially available plate reader with the associated software package (see Table of Materials). For using alternative instruments, refer to the sample preparation methods described by the instrument's manufacturer.

1. Dilute $20 \mu \mathrm{L}$ of NP solution with $80 \mu \mathrm{L}$ of ultrapure water (analysis concentration: $1 \mathrm{mg} / \mathrm{mL}$ PEG-PLA NPs). Add $30 \mu \mathrm{L}$ per well to a clear bottom black 384well plate (analyze in triplicate).

2. Measure the hydrodynamic radius and polydispersity of each sample with a DLS plate reader using preset protocol options in the software package. As an example of a typical protocol, set the data collection parameters to acquire 5-10 DLS measurements of 2-5 s duration per acquisition and then report a mean particle size and distribution per well, calculated from the globular proteins model. To form hydrogels with consistent rheological properties, the resulting particles should be $30-50$ $\mathrm{nm}$ in hydrodynamic diameter with a polydispersity $(\mathrm{PD})<0.2$.

NOTE: If the NPs are smaller than desired, use a solution of $75 \%$ acetonitrile / $25 \%$ dimethyl sulfoxide (DMSO) in step 1.1. Increasing the percentage of DMSO in the solvent solution will generally increase particle size.

5. Transfer the NP solution from the $20 \mathrm{~mL}$ scintillation vial into a centrifugal filter unit. Centrifuge at $4500 \times g$ for $1 \mathrm{~h}$ to concentrate the NP solution to $<250 \mu \mathrm{L}$.

6. Resuspend in desired buffer, such as phosphatebuffered saline (PBS), to $20 \mathrm{wt} \%$ NPs. Pipette the contents of the centrifugal filter unit into a tared microcentrifuge tube or glass scintillation vial on a mass balance. Use a small amount (50-100 $\mu \mathrm{L})$ of buffer to rinse the filter and ensure collection of all NPs. Add buffer to reach a total mass of $250 \mathrm{mg}$.

NOTE: Batches can be pooled during resuspension. NP stock solutions can be stored at $4{ }^{\circ} \mathrm{C}$ for approximately 1 month. Do not freeze. For longer storage, verify size and polydispersity by DLS before use.

\section{Hydrogel formulation and encapsulation of drugs or cells}

NOTE: This section describes preparation of $1 \mathrm{~mL}$ of 2:10 PNP hydrogel formulation, with 2:10 denoting $2 \mathrm{wt} \%$ HPMC$\mathrm{C}_{12}$ and $10 \mathrm{wt} \%$ NPs (12 wt $\%$ total solid polymer) and 88 
wt\% buffer solution, drug cargo solution, or cell suspension. The formulation percentages can be varied to yield hydrogels with a range of mechanical properties. For example, 1:5 PNP hydrogels were used for the cell settling and viability experimental results shown.

1. Prepare stock solution of 6 wt\% HPMC-C 12 in PBS (or other buffer of choice). Dissolve for $48 \mathrm{~h}$ to ensure the polymer is fully dispersed.

NOTE: The HPMC-C12 stock solution is stable for months at room temperature. However, storage at $4{ }^{\circ} \mathrm{C}$ is recommended to inhibit microbial growth.

2. Add $333 \mathrm{mg}$ of $6 \mathrm{wt} \% \mathrm{HPMC}-\mathrm{C}_{12}$ stock solution into a 1 mL Luer lock syringe.

3. Add $500 \mu \mathrm{L}$ of $20 \mathrm{wt} \% \mathrm{NP}$ stock solution to a microcentrifuge tube. Add $167 \mu \mathrm{L}$ of PBS and pipette to mix. Using a needle, fill another $1 \mathrm{~mL}$ Luer lock syringe with the diluted NP solution.

NOTE: To load drug cargo, calculate the desired final concentration of the drug in the hydrogel and load the appropriate amount into the $167 \mu \mathrm{L}$ of PBS that is mixed with the NPs. If a molecular probe is necessary for an in situ assay, such as for monitoring drug stability, load the probe in a similar manner as described above for loading drug cargo. To load cells, calculate the desired final cell concentration in the hydrogel and load the appropriate number of cells into the $167 \mu \mathrm{L}$ of PBS that is mixed with the NPs.

4. Mix the two hydrogel components (HPMC-C 12 and NPs) using an elbow mixing method ${ }^{35}$.

1. Attach the Luer elbow connector to the syringe containing NP solution (optionally also containing drug cargo or cells). Push the NP solution through the elbow until the meniscus is visible at the open end. Pull back slightly and connect the syringe containing HPMC-C 12 solution.

NOTE: It is important to minimize air in the elbow connection to prevent the formation and dispersal of bubbles throughout the hydrogel during the mixing process. When mixing cells with the elbow mixer, take care to mix more gently as mixing too rapidly may subject the cells to high shear forces, leading to cell death.

2. Pump the two solutions back and forth through the elbow mixer for approximately 60 cycles until a homogeneous, opaque white hydrogel material has formed.

3. Push the entire volume of hydrogel into one syringe. Remove the empty syringe and draw the plunger back on the gel-loaded syringe to recover material from the elbow connector. Cap with a needle or plug. NOTE: It is necessary to account for $\sim 300 \mu \mathrm{L}$ of lost hydrogel volume due to the dead space in the mixing process. For example, if $700 \mu \mathrm{L}$ of final hydrogel volume is desired, $1 \mathrm{~mL}$ of hydrogel should be prepared. The hydrogel formulation process can be scaled up by using larger syringes. However, for stiff hydrogel formulations, such as $2: 10$, it can become difficult to mix and inject from syringes larger than 3 $\mathrm{mL}$ in volume due to the increasing ratio of syringe barrel to elbow or needle diameter.

4. Store the hydrogel in the syringe at room temperature. However, if drugs are encapsulated, storage at $4{ }^{\circ} \mathrm{C}$ is recommended unless the drug manufacturer specifies otherwise. Do not freeze the material. 


\section{Measuring rheological properties of hydrogel formulations}

NOTE: This protocol is specifically used with the commercial rheometer mentioned in the Table of Materials with a $20 \mathrm{~mm}$ serrated plate geometry. For using other instruments, refer to the manufacturer's instructions for sample preparation.

1. Formulate at least $700 \mu \mathrm{L}$ of PNP hydrogel for rheological characterization.

2. Inject material in the center of the serrated rheometer plate. The amount will vary depending on the chosen geometry gap. For reference, a $700 \mu \mathrm{m}$ gap requires $\sim 400-500 \mu \mathrm{L}$ of material.

3. Lower the rheometer to the trim gap (500-1000 $\mu \mathrm{m})$ and slowly turn the top rheometer plate as it makes contact with the PNP hydrogel to ensure the gap is filled evenly and completely.

4. Inspect the loading of the PNP hydrogel such that it covers the entire rheometer plate surface. Use a spatula or plastic trimmer to gently trim and remove any excess material, such that it has a very slight bulge out of the plate.

5. Lower the rheometer to the final geometry gap and verify the sample is cleanly loaded.

6. Measure the mechanical properties of the sample using oscillatory tests, such as amplitude or frequency sweeps, or flow tests, such as flow sweeps or step tests.

NOTE: In the representative data shown, oscillatory amplitude tests are run at a constant frequency of $10 \mathrm{rad} / \mathrm{s}$. Oscillatory frequency sweeps are run at a constant $1 \%$ strain, within the linear viscoelastic regime of the amplitude sweep. Flow sweeps were run from high shear rates to low shear rates ${ }^{39}$. All tests are completed with 10 points per decade of data collected and at room temperature. The test parameters may need to be adjusted depending the properties of the formulation. Subjecting stiffer PNP materials such as $2: 10$ formulations to high shear rates can cause the material to be ejected from the rheometer plates, resulting in inaccurate mechanical characterization, and requiring reloading of the sample between subsequent tests. Representative data shown below can be used for comparison during quality control testing.

\section{Characterizing in vitro drug release}

1. Prepare capillary tubes by cutting glass capillary tubes to desired length. Seal one end of each tube by using a disposable spatula or pipette tip to push a small amount of epoxy into the end of the tube to form a plug. Allow epoxy to set per manufacturer's recommended time.

NOTE: The tube must be shorter than the length of the injection needle. Tubing with 2-3 $\mu \mathrm{m}$ inner diameter is recommended such that a length of 2.5 in will contain at least $300 \mu \mathrm{L}$ of total volume.

2. Formulate at least $500 \mu \mathrm{L}$ of a PNP hydrogel material in a syringe containing the drug of interest. Prepare each sample group in a separate syringe.

3. Inject $100-200 \mu \mathrm{L}$ of the PNP hydrogel at the bottom of each capillary tube using a long hypodermic needle (22G, 4 inch). Prepare at least three tubes (triplicate) per sample group.

4. (Optional) Place filled capillary tubes in a conical centrifuge tube and centrifuge for $1 \mathrm{~min}$ at $1000 \times \mathrm{g}$ to ensure the surface of the hydrogel is uniform. This step may need to be repeated, altering time and speed as necessary to smooth the surface of the material.

CAUTION: Ensure centrifuge is well balanced. 
5. Carefully fill $200-300 \mu \mathrm{L}$ of PBS on top of the hydrogel in the capillary tube using a syringe and needle or pipette. Do not contact or disturb the surface of the hydrogel. Seal the tube with a cap or plug or cover with at least two layers of paraffin film.

6. (Optional) Incubate samples at $37^{\circ} \mathrm{C}$ to simulate in vivo conditions.

7. Carefully completely remove the PBS from each capillary, without disturbing the hydrogel surface, using a syringe and needle at chosen time points depending on the anticipated time scale of drug release. Replace the volume removed with fresh PBS. Store aliquots under appropriate conditions.

NOTE: The recommended volumes and time points in steps $4.3,4.5$ and 4.7 can be optimized to capture in vitro drug release over a range of timescales, depending on how much drug is loaded in the material and how quickly it releases into the supernatant. A sample of selected time points could be 6 h, 1 day, 3 days, 1 week and 2 weeks for a slow-releasing drug. Aliquots can also be analyzed as they are acquired rather than stored.

8. At the completion of the study, analyze aliquots with an appropriate method such as ELISA, HPLC or fluorescence assay to quantify the amount of drug released at each time point $40,41,42$. The appropriate detection method will vary depending on the drug of interest.

NOTE: In vitro release studies are useful for comparing release between different hydrogel formulations or drug cargo. The in vitro release timescale does not often directly indicate an expected time scale of release in vivo.

\section{Characterizing thermal stability of gel- encapsulated insulin}

1. Formulate at least $1.2 \mathrm{~mL}$ of PNP hydrogel per sample group. Following the procedure described in section 2.3, load both insulin (drug cargo) and thioflavin T (ThT) (molecular probe) into the PNP hydrogel.

NOTE: The primary mechanism of aggregation and, therefore, inactivation of insulin is through the formation of amyloid fibrils. ThT is a suitable molecular probe because it produces a strong fluorescence signal in the presence of amyloid fibrils, allowing for in situ monitoring of insulin aggregation. Depending on the drug cargo of interest, aggregation may be monitored through different methods. For the representative data shown, insulin was loaded to a final concentration of 6.7 or $10 \mathrm{mg} / \mathrm{mL}$ and ThT to a final concentration of $25 \mu \mathrm{M}$.

2. Using a $21 \mathrm{G}$ needle, inject $200 \mu \mathrm{L}$ of PNP hydrogel per well into a black 96-well plate. Each sample group should be measured in at least triplicate. Seal plate with an optically clear adhesive plate seal to prevent evaporation.

3. Insert plate into a plate reader equipped with temperature control, shaking, and kinetic read programming and begin read protocol. Representative data was acquired with a commercially available plate reader (see Table of Materials) using the following conditions:

1. Stressed aging conditions: continuous linear shaking (410 cpm, $5 \mathrm{~mm})$ at $37^{\circ} \mathrm{C}$.

2. Data acquisition: excitation/emission $450 \mathrm{~nm} / 482$ $\mathrm{nm}$ at 20 min intervals

NOTE: If a plate reader with temperature control, shaker, and kinetic read capabilities is not available, the plate can be placed on a shaker plate in an 
incubator and read manually at above wavelengths at selected time points.

4. Plot data as mean fluorescence signal over time for each group. Time to aggregation can be quantified by defining an arbitrary signal threshold ${ }^{43}$.

NOTE: For the representative data shown below, the threshold was defined as 750,000 arbitrary fluorescence units (AFU). This value was chosen to be above the measured baseline while still sufficiently capturing the onset of aggregation indicated by a sharp fluorescent signal increase.

5. Terminate assay when samples aggregate or visually begin to dehydrate.

\section{Assessing cell viability}

1. Formulate at least $2 \mathrm{~mL}$ of PNP hydrogel containing the desired cell concentration following above protocols (normally $1-5 \times 10^{6}$ cells $/ \mathrm{mL}$ ). Prepare each sample group in a separate syringe.

2. Using a $21 \mathrm{G}$ needle, inject $150 \mu \mathrm{L}$ PNP hydrogel into each well in a clear bottom 96-well plate; each well is one replicate. Each sample group should have 3-5 replicates per time point. Centrifuge the plate at $50 \times g$ for $2 \mathrm{~min}$ to spread the hydrogel evenly in the well.

3. Add $100 \mu \mathrm{L}$ of the appropriate cell media on top of the hydrogel. Remove this media each day and add $100 \mu \mathrm{L}$ new media.

4. On Day 1, remove the media on top of the hydrogel for the designated replicates for that time point for each sample group. Add $50 \mu \mathrm{L}$ of $2 \mathrm{mM}$ calcein AM solution on top of the hydrogels. Incubate for $30 \mathrm{~min}$.

NOTE: Calcein AM can be used to identify and label live cells. In live cells, the non-fluorescent calcein AM is converted to a green-fluorescent calcein, by intracellular esterases after acetoxymethyl ester hydrolysis.

5. Image the center of each well in a 96-well plate using a confocal microscope. Survey an area of least 300 $\mu \mathrm{m}^{2}$ with a z-stack spanning at least $150 \mu \mathrm{m}$. Use confocal wavelength settings to capture the fluorescence of calcein (excitation/emission: $495 \mathrm{~nm} / 515 \mathrm{~nm}$ ).

6. Repeat step 6.4 and 6.5 for each subsequent time point as desired.

7. To analyze each image, collapse all z-stack images into a single plane maximum intensity image using FIJI or similar software. Quantify the number of fluorescent cells in each image. The ratio of the number of fluorescent cells at each time point compared to the number of fluorescent cells at Day 1 is the relative cell viability in the PNP hydrogels.

\section{Assessing cell settling}

1. Calculate the number of cells required to formulate 500-700 $\mu \mathrm{L}$ of PNP hydrogel at a final concentration of $5 \times 10^{6}$ cells $/ \mathrm{mL}$. Suspend cells in $1 \mathrm{~mL}$ of PBS at a concentration of $1 \times 10^{6}$ cells $/ \mathrm{mL}$. Stain cells by adding $50 \mu \mathrm{L}$ of $2 \mathrm{mM}$ calcein AM. Incubate the cells with the dye for $10 \mathrm{~min}$.

2. Centrifuge cells at appropriate conditions, remove the PBS and resuspend the cells in the volume of PBS needed to form $500-700 \mu \mathrm{L}$ of the desired PNP hydrogel. NOTE: The recommended speed and duration to centrifuge each specific cell type is typically provided in the product documentation.

3. Formulate $500-700 \mu \mathrm{L}$ of PNP hydrogel with the stained cells $\left(5 \times 10^{6}\right.$ cells $\left./ \mathrm{mL}\right)$ following protocol section 2 . 
4. Using a $21 \mathrm{G}$ needle, inject $100-200 \mu \mathrm{L}$ of PNP hydrogel containing the stained cells in the bottom of a cuvette. Three replicates should be performed for each sample. Move the needle back and forth within the cuvette while injecting to prevent bubble formation.

5. Immediately (time $t=0$ ), image the cuvettes lying on their side over the entire flat cuvette rectangle area at the base of the cuvette. Use the confocal tile scanning capabilities to image the entire well area and image a z-stack in 3D across a $100 \mu \mathrm{m}$ depth. For later visualization, either use the confocal microscope software to stitch together all of the individual tiles and perform a maximum intensity projection to form a single image of the large area or use FIJI software on a personal computer 44,45 .

6. Following imaging, stand the cuvettes upright.

7. Image at $1 \mathrm{~h}$ and $4 \mathrm{~h}$ to observe if cells have settled in the hydrogel or whether they remain suspended.

NOTE: These time points are suggestions and can be modified as desired.

8. To analyze each image, collapse all z-stack images into a single plane maximum intensity image. Using FIJI or similar software, quantify the cell distribution by measuring the fluorescence intensity down the center vertical profile of the cuvette to determine the degree of settling.

\section{Representative Results}

\section{PNP hydrogel fabrication and characterization}

PNP hydrogels are formed through the mixing of the two primary components - hydrophobically-modified HPMC polymers and PEG-PLA nanoparticles (Figure 1a). Therapeutic cargo is most easily incorporated into the additional buffer used to dilute the nanoparticle component prior to hydrogel preparation. For downstream biomedical characterization, it is convenient to use an elbow mixing method that enables simple and reproducible mixing of the two components (Figure 1b). After adequate mixing, the hydrogel should feel firm in the syringe, but yield under pressure and extrude from a standard needle (21G shown) (Figure 1c). After injection, the hydrogel should rapidly set into a solid-like material that resists flow from gravity. To fully characterize the hydrogel and ensure consistent batch-to-batch products, samples should be analyzed using several different experiments on a rheometer. The shearthinning and self-healing capabilities of the gel will be easily observed using a flow sweep protocol and step-shear protocol, respectively (Figure 2a,b). For stiffer gels, such as the 2:10 formulation, the user should look for viscosity to decrease at least two orders of magnitude during the flow sweep as the shear rate is increased from 0.1 to $100 \mathrm{~s}^{-1}$, which simulates the mechanical conditions during injection. The step-shear protocol should reveal an orders-ofmagnitude decrease in viscosity under the high-shear steps, and a rapid return ( $<5 \mathrm{~s}$ recovery time) to baseline viscosity during the low shear steps. Characterization of the storage and loss moduli using an oscillatory shear frequency sweep experiment in the linear viscoelastic regime should reveal solid-like properties at frequency ranges from 0.1-100 rad $\mathrm{s}^{-1}$ (Figure 2c). In particular, there should typically not be a crossover of the shear storage and loss moduli that is observable at low frequencies for stiffer formulations like the 2:10 hydrogels. Such a crossover event may indicate issues in the quality of the starting materials, either the modified HPMC or PEG-PLA polymer, or the size and dispersity of the PEG-PLA nanoparticles. It should be noted that a crossover event can be expected for weaker hydrogel formulations, such as the 1:5 hydrogel. Oscillatory shear amplitude sweeps on PNP hydrogels reveal that the materials do not yield until 
high stress values are applied, indicating these materials possess a yield stress, a threshold amount of stress required for the material to flow.

\section{Characterizing release kinetics from PNP hydrogels}

An essential step in designing PNP gels for drug delivery is the characterization of drug release kinetics from a chosen formulation. There are several techniques for this, but a simple in vitro methodology provides useful data during early formulation development (Figure 3a). Varying the polymer content of the PNP hydrogels through modulating the amount of HPMC-C 12 or NPs is the most straightforward way to tune the mechanical properties and mesh size of these hydrogels, which can have a direct impact on the diffusion of cargo through the polymer network and rate of release from the materials (Figure 3b). For cargo that is larger than the dynamic mesh size (i.e., high molecular weight or large hydrodynamic radius), researchers should expect a slow, dissolution-mediated release of cargo from the hydrogel depot. Formulations with dynamic mesh sizes greater than or equal to the size of the cargo will allow for diffusion-mediated release that can be described using traditional models of cargo diffusion and release $46,47,48,49$. Based on the shape of the release curve, researchers can reformulate the hydrogel to tune it towards slower (e.g., increase the polymer content) or faster (e.g., decrease the polymer content) release.

\section{Assessing stability of therapeutic cargo}

Determining the stability of the therapeutic cargo in a hydrogel formulation is critical before commencing preclinical or cellular studies. Compared to other synthetic methods for encapsulating drugs, PNP hydrogels incorporate cargo in a gentle manner by mixing into the bulk material, and it is unlikely that encapsulation will damage the cargo. These studies indicate that PNP hydrogels can also stabilize cargo that is susceptible to thermal instability, such as insulin, considerably extending shelf life and reducing reliance on cold storage and distribution (Figure 4). It is important to evaluate the condition of the cargo immediately after encapsulation into the hydrogel as well as after extended periods of storage. These data show that insulin remains stable in hydrogels after 28 days of storage under continuous thermal and mechanical stress, using a simple fluorescence assay for measuring insulin aggregation. An alternative technique for cases where an appropriate plate assay is unavailable would be to perform circular dichroism measurements of the cargo, which is particularly useful for determining the secondary structure of protein drugs.

\section{Determining cell viability and dispersion in PNP hydrogels}

Many therapeutic cells require adhesion motifs to remain viable, and thus inclusion of integrin motifs like arginineglycine-aspartic acid (RGD) peptides is an important step in adapting PNP hydrogels for cellular therapies ${ }^{50}$. The modular PEG-PLA polymer comprising the NPs enables chemical functionalization of the PEG corona through simple "click" chemistries ${ }^{28,51}$. In this example, cell-adhesive RGD peptides were attached to the PEG-PLA polymer to promote cell engagement with the PNP hydrogel structure. Formulations lacking adhesion sites will have low cell viability as encapsulated cells fail to proliferate compared to cells encapsulated in formulations with these adhesion motifs (Figure 5a,b). Encapsulated cells can be labeled with calcein AM or another appropriate fluorescent dye (e.g., CFSE) to facilitate cell counting with a fluorescence microscope. During optimization, viability should be compared to unmodified PNP hydrogels to assure integrin-functionalized formulations are providing enhanced viability and proliferation. If integrin- 
functionalized formulations are providing similar efficacy as unmodified hydrogels, this may indicate a failure in the conjugation chemistry used to incorporate the adhesion motifs.

Researchers should expect encapsulated cells to be evenly dispersed through the hydrogel medium when using an appropriate hydrogel formulation. This will allow for consistent and predictable dosing of cells during hydrogel administration and should translate to local retention of cells in the hydrogel after administration. The distribution of cells can be easily determined using fluorescence microscopy techniques. Cells can be labeled with an appropriate dye and then imaged using confocal microscopy. The images can be assessed visually (Figure 5c) and also quantitatively (Figure 5d) using ImageJ software to measure the average fluorescence intensity along the vertical axis of the image (or along whichever axis cell-settling due to gravity is expected to occur). If the hydrogel formulation is too weak to support the cells in suspension over prolonged timeframes, cell settling will occur, as observed in the 1:1 formulation in Figure 5. Increasing the polymer content can resolve issues with inhomogeneous cell dispersion due to settling. 
(a)
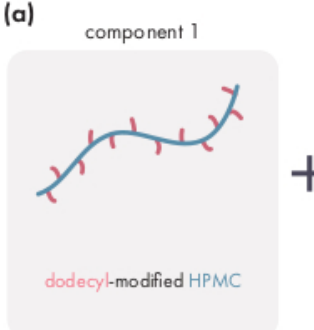

(b)

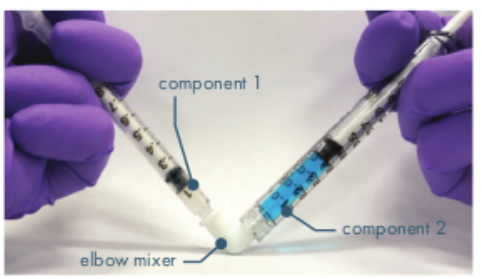

component 2

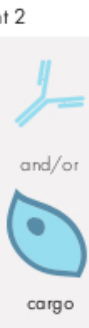

(c)

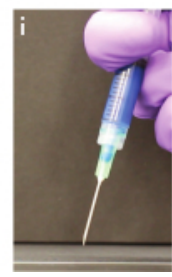

Injectable polymer-nanoparticle (PNP) hydrogels

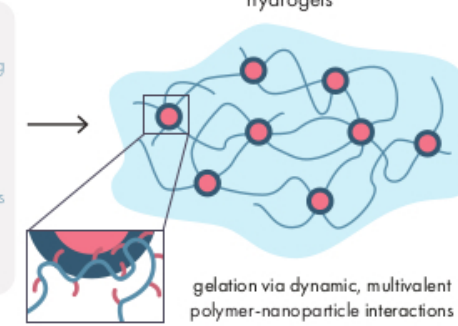

hydrogel injection through needles
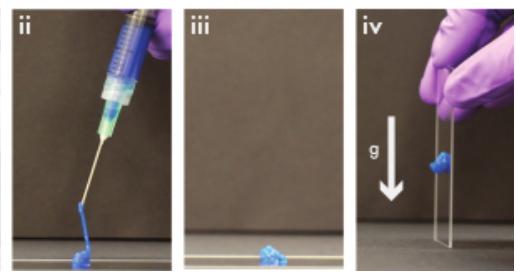

Figure 1: Polymer-nanoparticle (PNP) hydrogels are easily formed by mixing two components. (a) The first component is a solution of dodecyl-modified hydroxypropylmethyl cellulose (HPMC- ${ }_{12}$ ), and the second component is a solution of poly(ethylene glycol)-block-poly(lactic acid) (PEG-PLA) nanoparticles along with any therapeutic cargo. Gentle mixing of these two components yields an injectable hydrogel, where the HPMC- $\mathrm{C}_{12}$ polymers are physically crosslinked by dynamic, multivalent interactions with the PEG-PLA nanoparticles. (b) Photograph demonstrating gel formulation by mixing with two syringes, each one containing one component of the PNP hydrogel. By connecting the two syringes with a Luer-lock elbow connector, the two components can be easily mixed under sterile conditions to yield a bubble-free hydrogel pre-loaded into a syringe for immediate use. The NP solution is dyed blue for the purpose of demonstration. (c) Demonstration of the injection of PNP hydrogels and their re-solidification. (i) PNP hydrogel in a syringe with an attached 21G needle. (ii) Injection places the hydrogel under shear which temporarily breaks the interactions between polymer and nanoparticles, creating a fluid-like consistency. (iii) Post-injection, the dynamic polymer-nanoparticle interactions rapidly reform, allowing the hydrogel to self-heal into a solid. (iv) The solid hydrogel does not flow under forces weaker than its yield stress, such as gravity. The PNP hydrogel is dyed blue for the purpose of demonstration. Please click here to view a larger version of this figure. 


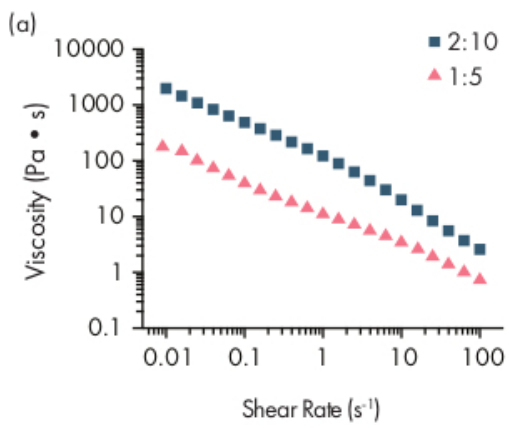

(b)
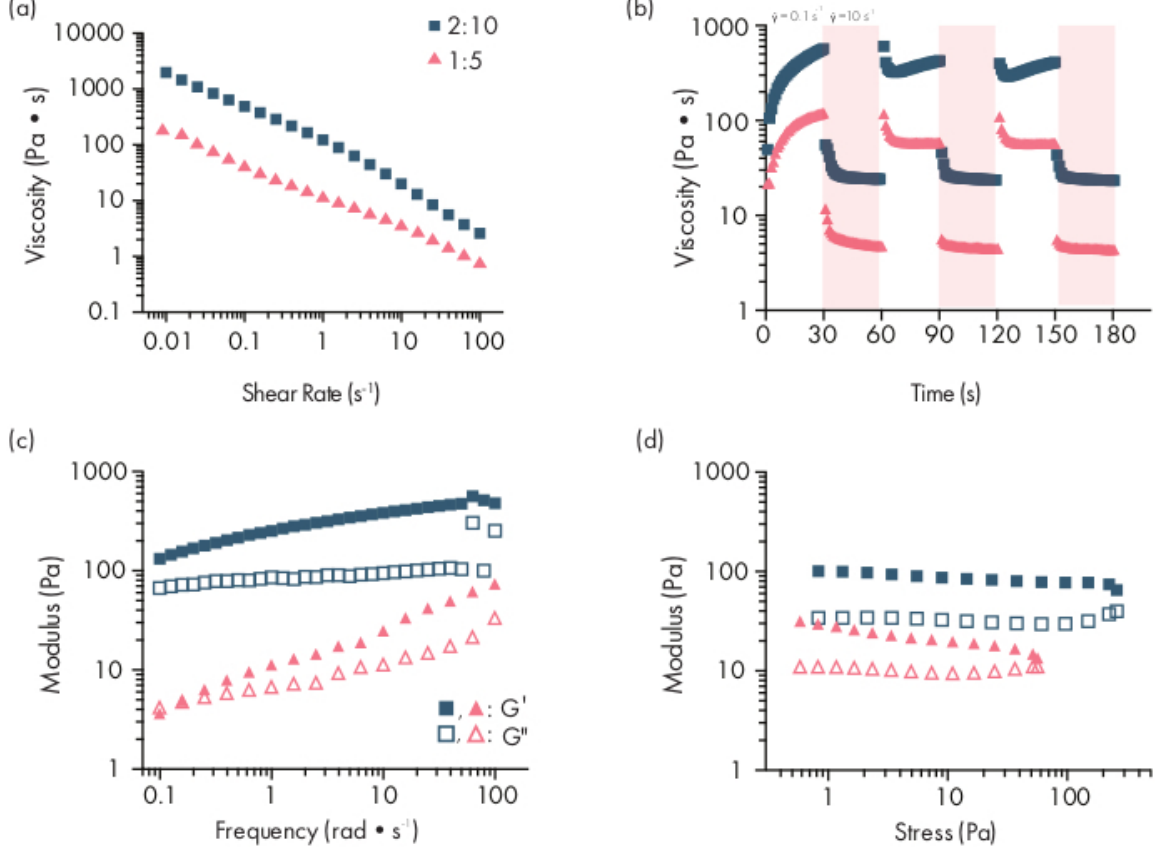

Figure 2: Rheological characterization of two PNP hydrogel formulations. Formulations are denoted as polymer wt. \%: NP wt.\%. (a) Steady shear flow sweeps from low to high shear rate of PNP hydrogels. Viscosity as a function of shear rate characterizes shear-thinning properties. (b) Viscosity as a function of oscillating shear rates between low shear rates (white background; $0.1 \mathrm{~s}^{-1}$ ) to high shear rates (red background; $10 \mathrm{~s}^{-1}$ ) demonstrating self-healing properties of PNP hydrogels. Shear rates are imposed for $30 \mathrm{~s}$ each. (c) Elastic storage modulus $G^{\prime}$ and viscous loss modulus $G$ " as a function of frequency at a constant $1 \%$ strain for various PNP hydrogel formulations. (d) Amplitude sweeps at a constant frequency of $10 \mathrm{rad} / \mathrm{s}$ to characterize elastic storage modulus $G^{\prime}$ and viscous loss modulus G" of PNP hydrogels as a function of stress. This rheological characterization can be used as comparison for quality control. This figure has been adapted from Grosskopf et al. ${ }^{28}$ Please click here to view a larger version of this figure. 
(a)

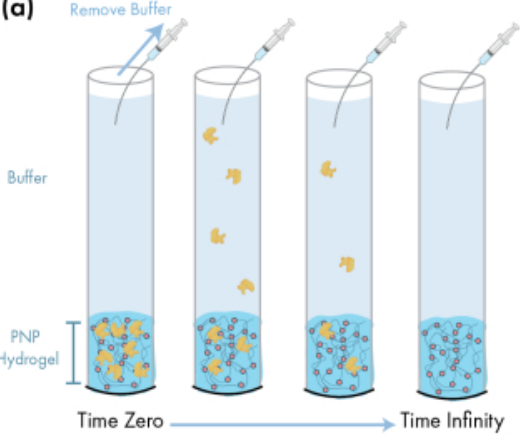

(b)

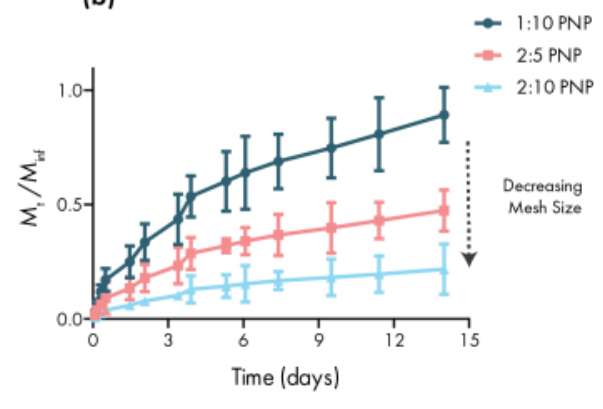

Figure 3: In vitro release of bovine serum albumin (BSA) from PNP hydrogels. Formulations are denoted as polymer wt. \%: NP wt.\%. (a) Schematic describing the experimental in vitro release protocol. Aliquots are removed from PNP hydrogelloaded capillary tubes over time. (b) The in vitro release of BSA from 1:10 PNP, 2:5 PNP and 2:10 PNP reported as the mass collected by the specified time point divided by the total mass collected during the assay (data shown as mean \pm SD; $n$ $=3)$. BSA was detected through absorbance measurements. Please click here to view a larger version of this figure. 


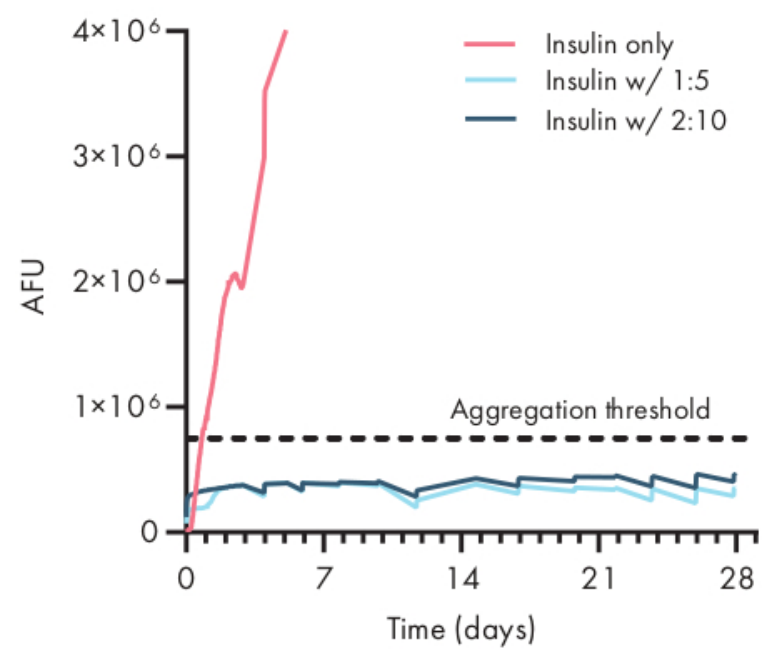

Figure 4: Thermal stability of insulin encapsulated in PNP hydrogels by ThT assay. Formulations are denoted as polymer wt.\%: NP wt.\%. Insulin encapsulated in both 1:5 and 2:10 PNP hydrogel remained unaggregated for over 28 days at stressed aging conditions of $37^{\circ} \mathrm{C}$ and constant agitation. Time to aggregation for insulin formulated in PBS was $20 \pm 4$ h (mean \pm SD, aggregation threshold 750,000 AFU). Data presented as an average of $n=4$ experimental replicates (AFU, arbitrary fluorescence units). This figure has been adapted from Meis et al. ${ }^{38}$ Please click here to view a larger version of this figure. 

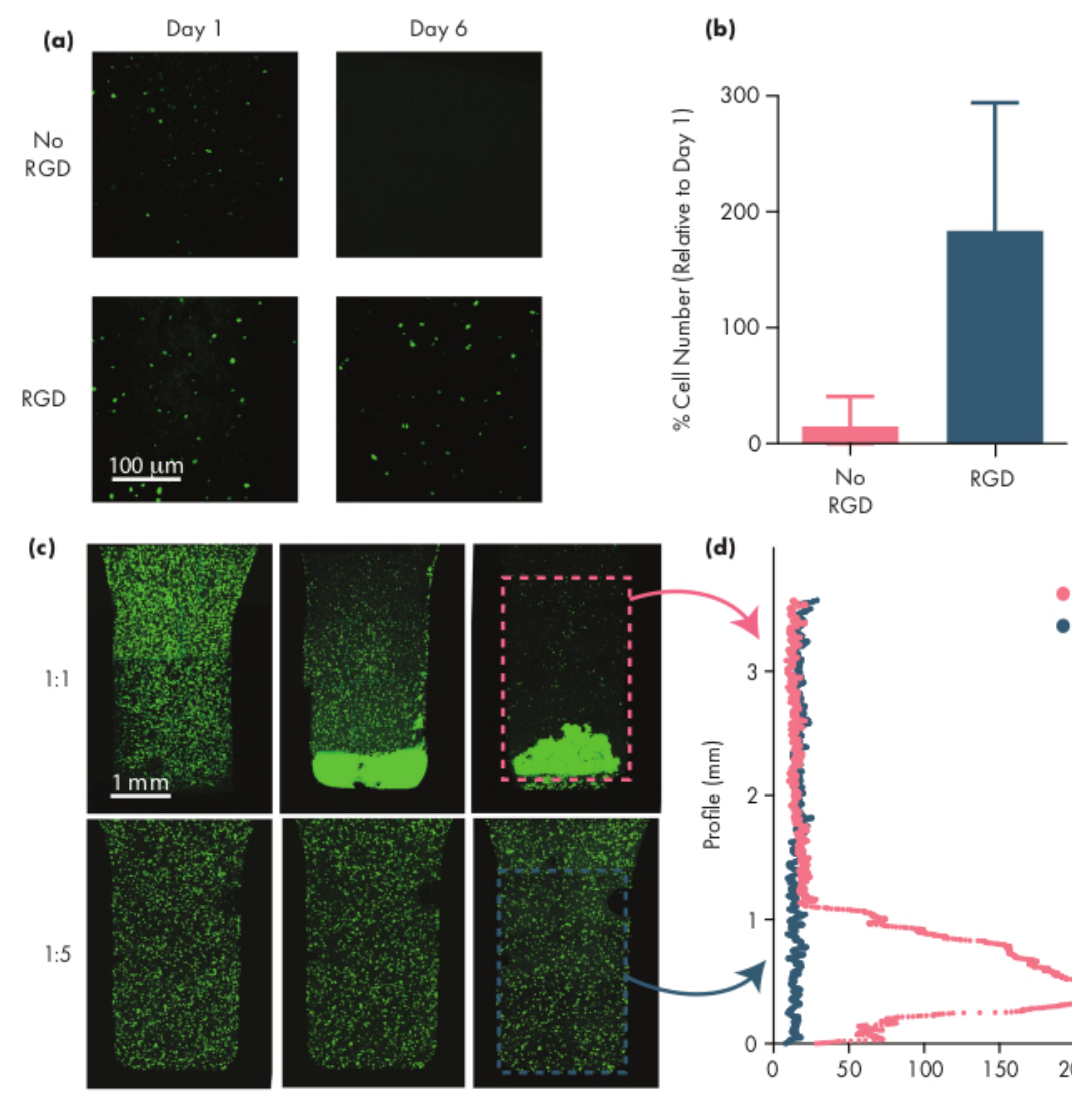

(d)

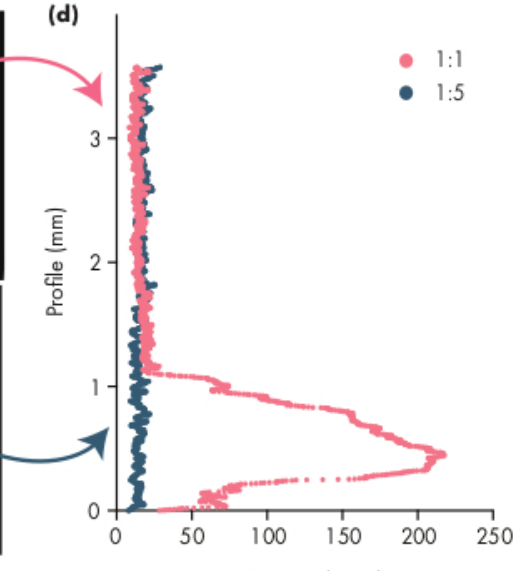

$15 \mathrm{~min}$

1 hour

4 hour

Average Horizontal Pixel Intensity

Figure 5: Cell viability and cell settling in PNP hydrogels. $(a, b)$ Cell viability studies in PNP hydrogels with human mesenchymal stem cells (hMSCs). (a) Representative images of viable hMSCs in 1:5 PNP hydrogels with and without the cell-adhesive arginine-glycine-aspartic acid (RGD) motif conjugated to the PEG-PLA NPs. hMSCs were calceinstained for $30 \mathrm{~min}$ prior to confocal imaging. Scale bar represents $100 \mu \mathrm{m}$. (b) Cell viability on Day 6 defined as number of fluorescent cells in the image relative to number of fluorescent cells on Day 1 (data shown as mean $\pm \mathrm{SD} ; n=3$ ). (c,d) Cell encapsulation and settling experiments with hMSCs. (c) Maximum intensity images of calcein AM-stained hMSCs encapsulated in 1:1 PNP hydrogel (top row) and 1:5 PNP hydrogel (bottom row) across $4 \mathrm{hr}$ to quantify cell settling. Scale bar represents $1 \mathrm{~mm}$. (d) Average horizontal pixel intensity of hMSCs along the vertical profile of the hydrogel. This figure has been adapted from Grosskopf et al. ${ }^{28}$ Please click here to view a larger version of this figure.

\section{Discussion}

Polymer-Nanoparticle (PNP) hydrogels are easily fabricated and enable the long-term local delivery of therapeutic cells and drugs through minimally invasive administration via direct injection or catheter delivery. These protocols describe the formulation of PNP hydrogels and the characterization methods for assuring quality of the resulting materials. Supramolecular PNP hydrogels are scalable to manufacture and are formed through the simple mixing of modified cellulose polymers and polymeric core-shell nanoparticles. 
The present methods describe facile procedures to form gels pre-loaded in syringes through simple elbow mixing protocols. Through quality control metrics of each of the component parts, such as DLS to monitor the NP size and distribution, one can reproducibly formulate PNP hydrogel materials with consistent rheological properties. Through varying the amount of HPMC-C 12 or NPs, one can modulate the mesh size and stiffness of the resulting PNP hydrogel. These properties can be tuned to best suit a particular biomedical application, and with the rheological methods detailed here researchers can characterize the shear-thinning and self-healing properties of PNP hydrogels as they optimize the platform for their specific applications. Methods for in vitro release studies are also described; researchers can use these studies to characterize the relative timescale of release of drugs of interest, informing future in vivo studies. Using stability studies, researchers can also assess the ability of these materials to help preserve the biological structure and stability of sensitive biotherapeutics over time and extreme temperatures, with compelling potential applications to reduce the cold chain dependence of biotherapeutics. Finally, with simple cell viability assays, cell growth and migration within PNP materials can be evaluated, with potential applications in cell therapies and scaffolds.

Our group has found many compelling applications for the PNP hydrogel platform ${ }^{27}$. PNP hydrogels have been used for slow delivery of subunit vaccines, enabling matched kinetic release profiles of antigens and adjuvants to boost the magnitude, duration, and quality of the humoral immune response $^{31}$. PNP hydrogels have been found to have a smaller mesh size than most commonly used hydrogels, so they are effective at slowing diffusion and slowly releasing molecular cargo. The unique tissue adherence properties and mechanical properties of PNP hydrogels have also been utilized to form physical barriers to prevent adhesions arising from surgery by spraying the hydrogels over large surface areas of organs following surgery ${ }^{30}$. PNP hydrogels have also been shown to be effective cell delivery vehicles, and the mechanical properties actually shield cells from the mechanical forces occurring in the syringe needle during injection, improving cell viability ${ }^{29}$. When the NPs are conjugated with a cell-adhesive peptide, cells can attach and engage with the PNP matrix to remain viable. Using this approach, PNP hydrogels have been shown to improve the local retention of injected stem cells compared to methods using liquid vehicles ${ }^{28}$. In addition, PNP hydrogels have been shown to prevent thermally-induced aggregation of encapsulated insulin, even under harsh stressed aging conditions, suggesting that these materials may be able to reduce the need to refrigerate temperature-sensitive drugs ${ }^{38}$.

Overall, the methodologies described here will allow research groups to fabricate and explore PNP hydrogels as a biomaterial. These protocols provide the lab-scale synthesis techniques to fabricate enough hydrogel material to pursue both in vitro and in vivo studies. The studies described above indicate that the dynamic crosslinks of these materials enable it to be suitable for a range of biomedical applications by allowing active motility of entrapped cells while restricting passive diffusion of molecular cargo. It is anticipated that researchers will find the PNP platform an accessible and powerful tool to improve clinical outcomes through controlled drug delivery and to study basic biological mechanisms such as cell recruitment and mechanobiology.

\section{Disclosures}

These authors have nothing to disclose.

\section{Acknowledgments}


This research was financially supported by the Center for Human Systems Immunology with Bill \& Melinda Gates Foundation (OPP1113682) and the Bill \& Melinda Gates Foundation (OPP1211043). C.M.M. was supported by a Stanford Graduate Fellowship and the Stanford Bio-X William and Lynda Steere Fellowship. A.K.G. is thankful for a National Science Foundation Graduate Research Fellowship and the Gabilan Fellowship of the Stanford Graduate Fellowship in Science and Engineering. S.C. was supported by the National Cancer Institute of the National Institutes of Health under Award Number F32CA247352. The authors would also like to warmly acknowledge Appel Lab members including Dr. Gillie Roth, Dr. Anthony Yu, Dr. Lyndsay Stapleton, Dr. Hector Lopez Hernandez, Dr. Andrea d'Aquino, Dr. Julie Baillet, Celine Liong, Ben Ou, Emily Meany, Emily Gale and Dr. Anton Smith for their effort and time in helping the Appel Lab to develop these protocols over the years.

\section{References}

1. Mandal, A., Clegg, J. R., Anselmo, A. C., Mitragotri, S. Hydrogels in the clinic. Bioengineering Translational Medicine. 5 (2), e10158 (2020).

2. Appel, E. A., del Barrio, J., Loh, X. J., Scherman, O. A. Supramolecular polymeric hydrogels. Chemical Society Reviews. 41 (18), 6195-6214 (2012).

3. Mann, J. L. Yu, A. C. Agmon, G., Appel, E. A. Supramolecular polymeric biomaterials. Biomaterials Science. 6 (1), 10-37, (2018).

4. Foster, A. A., Marquardt, L. M., Heilshorn, S. C. The diverse roles of hydrogel mechanics in injectable stem cell transplantation. Current Opinion in Chemical Engineering. 15, 15-23 (2017).
5. Aguado, B. A., Mulyasasmita, W., Su, J., Lampe, K. J., Heilshorn, S. C. Improving viability of stem cells during syringe needle flow through the design of hydrogel cell carriers. Tissue Engineering Part A. 18 (7-8), 806-815 (2012).

6. Marquardt, L. M., Heilshorn, S. C. Design of injectable materials to improve stem cell transplantation. Current Stem Cell Reports. 2 (3), 207-220 (2016).

7. Guvendiren, M., Burdick, J. A. Engineering synthetic hydrogel microenvironments to instruct stem cells. Current Opinion in Biotechnology. 24 (5), 841-846 (2013).

8. Marquardt, L. M. et al. Designer, injectable gels to prevent transplanted Schwann cell loss during spinal cord injury therapy. Science Advances. 6 (14), eaaz1039 (2020).

9. Stephan, S. B. et al. Biopolymer implants enhance the efficacy of adoptive T-cell therapy. Nature Biotechnology. 33 (1), 97-101 (2015).

10. Tuladhar, A. et al. Injectable hydrogel enables local and sustained co-delivery to the brain: two clinically approved biomolecules, cyclosporine and erythropoietin, accelerate functional recovery in rat model of stroke. Biomaterials. 235, 119794 (2020).

11. Pakulska, M. M., Miersch, S., Shoichet, M. S. Designer protein delivery: From natural to engineered affinitycontrolled release systems. Science. 351 (6279), (2016).

12. Gupta, D., Tator, C. H., Shoichet, M. S. Fast-gelling injectable blend of hyaluronan and methylcellulose for intrathecal, localized delivery to the injured spinal cord. Biomaterials. 27 (11), 2370-2379 (2006). 
13. Verbeke, C. S., Mooney, D. J. Injectable, pore-forming hydrogels for in vivo enrichment of immature dendritic cells. Advanced Healthcare Materials. 4 (17), 2677-2687 (2015).

14. Tous, E., Purcell, B., Ifkovits, J. L., Burdick, J. A. Injectable acellular hydrogels for cardiac repair. Journal of Cardiovascular Translational Research. 4 (5), 528-542 (2011).

15. Zhao, X. et al. Antibacterial anti-oxidant electroactive injectable hydrogel as self-healing wound dressing with hemostasis and adhesiveness for cutaneous wound healing. Biomaterials. 122, 34-47 (2017).

16. Johnson, T. D., Christman, K. L. Injectable hydrogel therapies and their delivery strategies for treating myocardial infarction. Expert Opinion on Drug Delivery. 10 (1), 59-72 (2013).

17. Kleinman, H. K., Martin, G. R. in Seminars in Cancer Biology. 378-386. Elsevier. (2005).

18. Hickey, J. W. et al. Engineering an artificial Tcell stimulating matrix for immunotherapy. Advanced Materials. 31 (23), 1807359 (2019).

19. Baumann, M. D. et al. An injectable drug delivery platform for sustained combination therapy. Journal of Controlled Release. 138 (3), 205-213 (2009).

20. Trappmann, B. et al. Matrix degradability controls multicellularity of $3 \mathrm{D}$ cell migration. Nature Communications. 8 (1), 1-8 (2017).

21. Figueiredo, L. et al. Assessing glucose and oxygen diffusion in hydrogels for the rational design of $3 \mathrm{D}$ stem cell scaffolds in regenerative medicine. Journal of Tissue Engineering and Regenerative Medicine. 12 (5), 1238-1246 (2018).
22. Daly, A. C., Riley, L., Segura, T., Burdick, J. A. Hydrogel microparticles for biomedical applications. Nature Reviews Materials. 1-24 (2019).

23. Chaudhuri, O. et al. Substrate stress relaxation regulates cell spreading. Nature Communications. 6 (1), 1-7 (2015).

24. Cai, L., Dewi, R. E., Heilshorn, S. C. Injectable hydrogels with in situ double network formation enhance retention of transplanted stem cells. Advanced Functional Materials. 25 (9), 1344-1351 (2015).

25. Fisher, S. A., Baker, A. E., Shoichet, M. S. Designing peptide and protein modified hydrogels: selecting the optimal conjugation strategy. Journal of the American Chemical Society. 139 (22), 7416-7427 (2017).

26. Li, R. H., Altreuter, D. H., Gentile, F. T. Transport characterization of hydrogel matrices for cell encapsulation. Biotechnology and Bioengineering. 50 (4), 365-373 (1996).

27. Appel, E. A. et al. Self-assembled hydrogels utilizing polymer-nanoparticle interactions. Nature Communications. 6 (6295), (2015).

28. Grosskopf, A. K. et al. Injectable supramolecular polymer-nanoparticle hydrogels enhance human mesenchymal stem cell delivery. Bioengineering and Translational Medicine. 5 (1), e10147 (2020).

29. Lopez Hernandez, H., Grosskopf, A. K., Stapleton, L. M., Agmon, G., Appel, E. A. Non-newtonian polymernanoparticle hydrogels enhance cell viability during injection. Macromolecular Bioscience. 19 (1), (2019).

30. Stapleton, L. M. et al. Use of a supramolecular polymeric hydrogel as an effective post-operative pericardial 
adhesion barrier. Nature Biomedical Engineering. 3 (8), 611-620 (2019).

31. Roth, G. A. et al. Injectable hydrogels for sustained codelivery of subunit vaccines enhance humoral immunity. ACS Central Science. (2020).

32. Steele, A. N. et al. A biocompatible therapeutic catheterdeliverable hydrogel for in situ tissue engineering. Advanced Healthcare Materials. 8 (5), 1801147 (2019).

33. Fenton, O. S. et al. Injectable polymer-nanoparticle hydrogels for local immune cell recruitment. Biomacromolecules. 20 (12), 4430-4436 (2019).

34. Yu, A. C., Smith, A. A., Appel, E. A. Structural considerations for physical hydrogels based on polymernanoparticle interactions. Molecular Systems Design \& Engineering. 5 (1), 401-407 (2020).

35. Wisdom, K., Chaudhuri, O. in 3D Cell Culture: Methods and Protocols. Editor: Zuzana Koledova) 29-37, Springer New York. (2017).

36. Lohmeijer, B. G. et al. Guanidine and amidine organocatalysts for ring-opening polymerization of cyclic esters. Macromolecules. 39 (25), 8574-8583 (2006).

37. Cheng, J. et al. Formulation of functionalized PLGAPEG nanoparticles for in vivo targeted drug delivery. Biomaterials. 28 (5), 869-876 (2007).

38. Meis, C. M. et al. Self-assembled, dilution-responsive hydrogels for enhanced thermal stability of insulin biopharmaceuticals. ACS Biomaterials Science \& Engineering. (2020).

39. Franck, A., Germany, T. Viscoelasticity and dynamic mechanical testing. TA Instruments, New Castle, DE, USA ANO04. (1993).
40. Appel, E. A. et al. Self-assembled hydrogels utilizing polymer-nanoparticle interactions. Nature Communications. 6, 6295 (2015).

41. Gallastegui, A. et al. Controlled release of antibiotics from photopolymerized hydrogels: kinetics and microbiological studies. Materials Science and Engineering: C. 102, 896-905 (2019).

42. Qiao, M., Chen, D., Ma, X., Liu, Y. Injectable biodegradable temperature-responsive PLGA-PEGPLGA copolymers: synthesis and effect of copolymer composition on the drug release from the copolymer-based hydrogels. International Journal of Pharmaceutics. 294 (1-2), 103-112 (2005).

43. Schlein, M. Insulin Formulation Characterization-The Thioflavin T Assays. The AAPS Journal. 19 (2), 397-408 (2017).

44. Preibisch, S., Saalfeld, S., Tomancak, P. Globally optimal stitching of tiled 3D microscopic image acquisitions. Bioinformatics. 25 (11), 1463-1465 (2009).

45. Sakas, G., Grimm, M., Savopoulos, A. in EUROGRAPHICS workshop on Rendering Techniques. 51-63, Springer. (1995).

46. Axpe, E. et al. A multiscale model for solute diffusion in hydrogels. Macromolecules. 52 (18), 6889-6897 (2019).

47. Peppas, N., Bures, P., Leobandung, W., Ichikawa, H. Hydrogels in pharmaceutical formulations. European Journal of Pharmaceutics and Biopharmaceutics. 50 (1), 27-46 (2000).

48. Ritger, P. L., Peppas, N. A. A simple equation for description of solute release I. Fickian and non-fickian release from non-swellable devices in the form of 
slabs, spheres, cylinders or discs. Journal of Controlled

Release. 5 (1), 23-36 (1987).

49. Reinhart, C. T., Peppas, N. A. Solute diffusion in swollen membranes. Part II. Influence of crosslinking on diffusive properties. Journal of Membrane Science. 18, 227-239 (1984).

50. Salinas, C. N., Anseth, K. S. The influence of the RGD peptide motif and its contextual presentation in PEG gels on human mesenchymal stem cell viability. Journal of Tissue Engineering and Regenerative Medicine. 2 (5), 296-304 (2008).

51. Smith, A. A. et al. Nanoparticles presenting potent TLR7/8 agonists enhance anti-PD-L1 immunotherapy in cancer treatment. Biomacromolecules. 21 (9), 3704-3712 (2020). 